\title{
Modeling of Profile Effects for Inductive Helicon Plasma Sources
}

\author{
Yiannis Mouzouris and John E. Scharer, Member, IEEE
}

\begin{abstract}
A computer code for modeling existing and new helicon sources for materials processing has been developed. The Nagoya type-III, helical, and Stix coil antennas have been modeled to study and examine plasma density and temperature profile effects on power absorption of a small fraction $\left(n_{f e} / n_{e} \approx\right.$ $5 \%$ ) of fast electrons ( $T_{\mathrm{e}-\text { fast }} \approx 40 \mathrm{eV}$ ) which provide ionization of the neutral gas in the experiment, and bulk $\left(T_{\text {e-slow }} \approx 3 \mathrm{eV}\right)$ electron distributions in an argon gas. The "ANTENA" computer code, originally written by $B$. McVey to study ion cyclotron waves, was modified and used to study and model helicon sources. A collisional model that includes radial density and temperature profiles was added to the code to study the effect of collisions on the heating mechanisms. The competing effects of collisional and Landau damping heating mechanisms have been investigated in detail, and results indicate that collisions play an important role in the plasma absorption profile at high densities $\left(n_{e} \geq 10^{13} \mathrm{~cm}^{-3}\right)$. The radio frequency wave absorption profiles are sensitive to the plasma density and temperature profiles. The partial-turn helix antenna, that solely excites the $m=+1$ azimuthal mode, is found to be more efficient in coupling the power to an assumed plasma profile than the Nagoya type-III. The Stix coil is also found to be promising due to its on-axis peaking of the wave heating fields.
\end{abstract}

\section{INTRODUCTION}

$\mathbf{P}$ LASMA etching has been one of the key technologies since 1970 [1] for the fabrication of semiconductor integrated circuits and microelectronic devices. A recent review paper [2] shows that the conventional capacitively coupled $\mathrm{RF}$ discharge plasma source does not appear to satisfy all the etching requirements for the ultra large scale integration (ULSI) ( $>10^{6}$ devices/circuit) technology. Therefore, it is necessary to search for other plasma sources. Electron cyclotron resonance (ECR), inductively coupled planar, and helicon sources are examples of plasma sources that can overcome the limitations of the RF capacitively coupled discharges. These sources operate at low pressures ( $\leq 5$ mtorr) (allowing anisotropic etching), high densities (higher etch rates), and provide reasonable uniformity. Helicon sources, which have a higher ionization efficiency and flux density, are easier to operate due to their higher radiation resistance and readily matched input impedance. They can sometimes be operated in weaker magnetic fields $\left(B_{0} \approx 50-100 \mathrm{G}\right)$ than resonant ECR sources $\left(B_{0} \approx 1 \mathrm{kG}\right.$ ). Magnetic fields up to $2 \mathrm{kG}$ have been employed in experiments [3]-[6] for fundamental helicon plasma source and wave studies.

Manuscript received January 30, 1995; revised November 24, 1995. This work was supported by the Graduate School of the University of WisconsinMadison, and in part by AFOSR Grant F49620-94-1-0054.

The authors are with the Department of Electrical and Computer Engineering, University of Wisconsin, Madison, WI 53706 USA.

Publisher Item Identifier S 0093-3813(96)02193-5.
In 1970, Boswell [7] found that a high-density plasma could be produced by the excitation of helicon waves in a cylindrical plasma. Experiments done by Boswell and his group show that peak densities on the order of $4 \times 10^{12} \mathrm{~cm}^{-3}$ in an argon gas could be produced with only $600 \mathrm{~W}$ of $8 \mathrm{MHz}$ power in a 5$\mathrm{cm}$ radius tube. Shoji et al. [6] used helical coils to excite the $m=+1$ or $m=-1$ azimuthal mode separately, depending on the direction of the static magnetic field. They obtained a higher plasma density $\left(10^{13} \mathrm{~cm}^{-3}\right)$ with the $m=+1$ mode than with the $m=-1$ mode.

Chen [8] has suggested Landau damping of the helicon wave as a possible mechanism for efficient energy transfer between the fast ionizing electrons and the wave. Experimental evidence of Landau damping on bulk slow electrons and primary fast electrons at lower densities has been presented by Komori [4], Chen [5], and Loewenhardt [9], [10]. Komori also showed that collisional damping is significant at high densities $\left(n_{e} \geq 7 \times 10^{12} \mathrm{~cm}^{-3}\right)$ and plays an important role in transferring the energy to the electrons. In particular, he shows that both Landau and collisional damping processes are important in the density range of $n_{e} \approx 10^{12}-3 \times 10^{13} \mathrm{~cm}^{-3}$.

In this paper, modeling of helicon sources has been studied with the aid of the computer code "ANTENA" [11], [12] that models the radio frequency antenna-plasma interaction. This code calculates the three-dimensional (3-D) electromagnetic plasma fields in a one-dimensional (1-D) cylindrical hot magnetized plasma surrounded by an RF inductive coil. We have modified this code to study and analyze helical antennas, along with the Nagoya type-III and $m=0$ Stix coil, for the plasma absorption profile of high-density plasma sources in the lower hybrid frequency range. These antennas can couple into a helicon mode wave propagating at frequencies near the lower hybrid frequency. We examine cases where the frequency is in the neighborhood of the lower hybrid frequency with an objective of obtaining a broad core heating profile. The "ANTENA" code treats the bulk fast and slow waves that propagate in the column subject to the inductive antenna spectrum and radial plasma boundary conditions. We will focus and study wave-particle physics that take place in the source region, where the RF power is coupled from the antenna to the plasma. In this region, the magnetic field is assumed to be uniform, and therefore, the code can be applied to accurately describe the antenna-plasma coupling.

An introduction to the physics of the "ANTENA" code, as well as the spatial current distribution of the modeled antennas, is presented in Section II. In Section III, we discuss modeling results of existing and new helicon sources, obtained from the "ANTENA" code. Section IV describes the conclusions. 


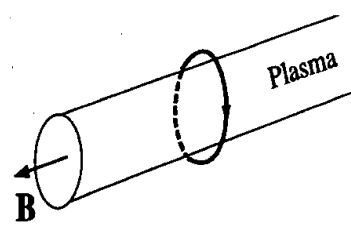

Full Turn Loop

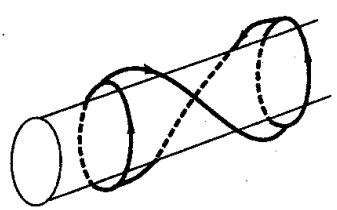

Fractional Helix

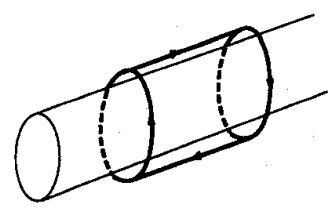

Nagoya Type-III

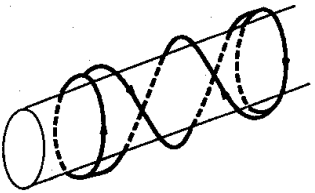

Integral Helix (1 turn)
Fig. 1. Various antenna configurations.

\section{INTRODUCTION TO THE PHYSICS OF THE “ANTENA" CODE}

The computer code "ANTENA" calculates the vacuum fields as well as the linear, inductive, self-consistent 3-D electromagnetic plasma fields for a variety of antenna configurations as shown in Fig. 1. It also has the capability to compute the radial power deposition profiles, the radial power flow, and the antenna impedance (resistance and reactance). Fig. 2 illustrates the antenna-plasma geometry. A cylindricalhot magnetized plasma is surrounded by an RF inductive coil, and both are enclosed in a metal conducting tube. The uniform magnetic field is oriented in the $z$-direction. The plasma density, $n_{e}(r)$, and plasma temperature, $T_{e}(r)$, are functions of radius and their radial variation is approximated by a stratified model. The plasma response is characterized by the plasma equivalent dielectric tensor [13], which includes selfconsistent Landau and collisional damping of the RF fields. A Maxwellian velocity distribution is assumed for both electrons and ions. The particle conserving Krook [14] collisional model implemented in "ANTENA" is introduced to model electron collisions in a phenomenological manner.

The geometry shown in Fig. 2, with the antennas removed, is uniform in the $z$ (axial) and $\phi$ (azimuthal) directions. Therefore, the spatial variation of field quantities in these directions can be expressed as a Fourier representation. The inverse Fourier transform is given by

$$
F(r, \phi, z)=\frac{1}{2 \pi} \int_{-\infty}^{+\infty} d k_{z} \sum_{m=-\infty}^{\infty} F\left(r, m, k_{z}\right) e^{i m \phi+i k_{z} z}
$$

and the Fourier coefficients can be written as

$$
F\left(r, m, k_{z}\right)=\int_{-\infty}^{+\infty} d z \frac{1}{2 \pi} \int_{0}^{2 \pi} d \phi F(r, \phi, z) e^{-i m \phi-i k_{z} z}
$$

Applying the Fourier transform to Maxwell's equations reduces the 3-D boundary value problem to a 1-D radial boundary value problem for each Fourier mode $\left(m, k_{z}\right)$. The real space field solutions are then obtained by summing over

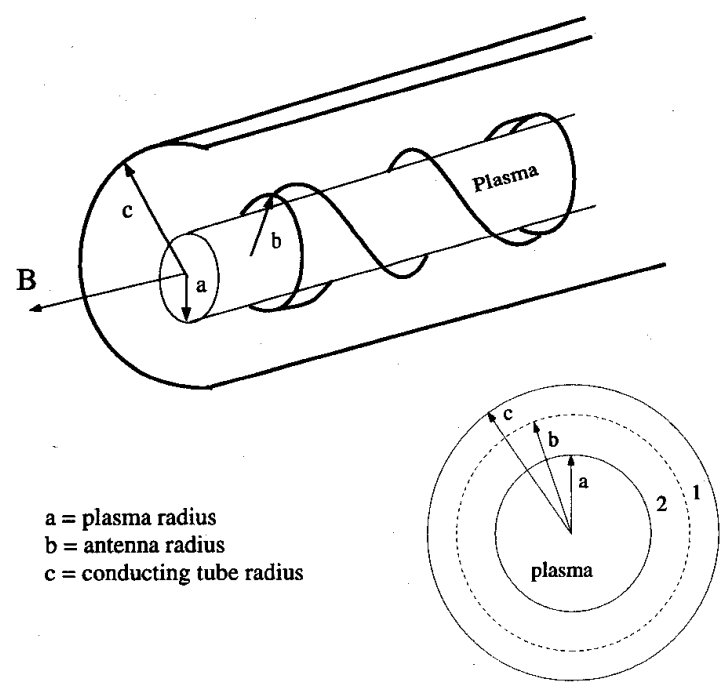

Fig. 2. Antenna-plasma geometry.

the Fourier modes (1). The current distribution on the antennas at $r=b$ is represented by an infinite set of current sheets with the complex exponential form $e^{i m \phi+i k_{z} z}$. The transformation of this current pattern to wave number $\left(m-k_{z}\right)$ space will determine the excitation level of a particular Fourier coefficient.

The vacuum electromagnetic fields in a cylindrical tube can be expanded into waveguide modes that are transverse electric (TE) and transverse magnetic (TM) to the axis of the cylindrical waveguide. The axial field components satisfy Bessel's equation

$$
\frac{d^{2}}{d r^{2}}\left\{\begin{array}{l}
H_{z} \\
E_{z}
\end{array}\right\}+\frac{1}{r} \frac{d}{d r}\left\{\begin{array}{l}
H_{z} \\
E_{z}
\end{array}\right\}-\left(\nu^{2}+\frac{m^{2}}{r^{2}}\right)\left\{\begin{array}{l}
H_{z} \\
E_{z}
\end{array}\right\}=0
$$

for a radial wave number given by

$$
\nu^{2}=k_{z}^{2}-k_{0}^{2}=k_{z}^{2}-\frac{\omega^{2}}{c^{2}} .
$$

The transverse fields are determined from Maxwell's equations in terms of the axial fields. In the plasma, Bessel's equation for the nearly TE modes becomes [12]

$$
\begin{aligned}
\frac{d^{2}}{d r^{2}} H_{z 1}+\frac{1}{r} & \frac{d}{d r} H_{z 1}+\left(k_{r 1}^{2}-\frac{m^{2}}{r^{2}}\right) H_{z 1}=0 \\
E_{z 1} & =z_{1} H_{z 1} \\
z_{1} & =\frac{i \omega \mu_{0} k_{z} D}{P\left(k_{z}^{2}-k_{0}^{2} S\right)+k_{r 1}^{2} S} .
\end{aligned}
$$

For the nearly TM modes we have

$$
\begin{aligned}
\frac{d^{2}}{d r^{2}} E_{z 2}+\frac{1}{r} & \frac{d}{d r} E_{z 2}+\left(k_{r 2}^{2}-\frac{m^{2}}{r^{2}}\right) E_{z 2}=0 \\
H_{z 2} & =y_{2} E_{z 2} \\
y_{2} & =\frac{-i \omega \epsilon_{0} k_{z} P D}{S\left(k_{r 2}^{2}+k_{z}^{2}\right)-R L k_{0}^{2}} .
\end{aligned}
$$


The radial wavenumbers $k_{r 1}, k_{r 2}$ are defined by

$$
\begin{aligned}
S k_{r i}^{4} & +k_{r i}^{2}\left[k_{z}^{2}(S+P)-k_{0}^{2}(S P+R L)\right] \\
& +P\left(k_{z}^{2}-k_{0}^{2}\right)\left(k_{z}^{2}-k_{0}^{2} L\right)=0 .
\end{aligned}
$$

The plasma is represented by the well-known elements of the "Stix" equivalent dielectric tensor [13]

$$
\vec{\epsilon}=\left[\begin{array}{ccc}
S & -i D & 0 \\
i D & S & 0 \\
0 & 0 & P
\end{array}\right]
$$

where the component $P$ includes both collisional and Landau damping effects, and is given by

$$
\begin{aligned}
P & =1-\sum_{\alpha} \frac{\omega_{p \alpha}^{2}}{\left(k_{z} v_{\alpha}\right)^{2}} \frac{Z^{\prime}\left(\zeta_{0}\right)}{1+\frac{i \nu_{\alpha}}{k_{z} v_{\alpha}} Z\left(\zeta_{0}\right)} \\
\zeta_{n} & =\frac{\omega+i \nu_{\alpha}+n \omega_{c \alpha}}{k_{z} v_{\alpha}}
\end{aligned}
$$

where $Z$ is the plasma dispersion function tabulated by Fried and Conte [15]

$$
Z(\zeta)=\frac{1}{\sqrt{\pi}} \int_{\infty}^{\infty} d z \frac{e^{-z^{2}}}{z-\zeta}, \quad \operatorname{Im} \zeta>0
$$

and

$$
\begin{aligned}
\alpha & =\text { species (electrons, ions, etc.), } \\
\omega_{p \alpha} & =\sqrt{\frac{n_{\alpha} e^{2}}{m_{\alpha} \epsilon_{0}}}=\text { plasma frequency, } \\
\omega_{c \alpha} & =\frac{q_{\alpha} B}{m_{\alpha}}=\text { cyclotron frequency, } \\
v_{\alpha} & =\sqrt{\frac{2 k T_{\alpha}}{m_{\alpha}}}=\text { thermal velocity, } \\
\nu_{\alpha} & =\text { collision frequency for species } \alpha .
\end{aligned}
$$

Returning to (1), the smaller root $\left(k_{r 1}\right)$ is assigned to the nearly transverse electric modes and the larger root $\left(k_{r 2}\right)$ to the nearly transverse magnetic modes. The total axial plasma fields are given by

$$
\begin{aligned}
& H_{z}=H_{z 1}+y_{2} E_{z 2} \\
& E_{z}=z_{1} H_{z 1}+E_{z 2} .
\end{aligned}
$$

The axial components $H_{z 1}(r)$ and $E_{z 2}(r)$ each separately satisfy Bessel's equation, and the transverse plasma fields $E_{r}, E_{\phi}, H_{r}, H_{\phi}$ are determined from the axial components. The above general solutions are solved by imposing boundary conditions at the interface between the three regions shown in Fig. 2.

At the conducting tube radius $r=c$, the tangential components of the electric field must vanish

$$
E_{\phi}=E_{z}=0 \text {. }
$$

Across the plasma-vacuum boundary at $r=a$, the tangential components of the electric and magnetic fields are continuous. That is

$$
\begin{array}{cc}
E_{\phi}^{v}=E_{\phi}^{p}, & E_{z}^{v}=E_{z}^{p} \\
H_{\phi}^{v}=H_{\phi}^{p}, & H_{z}^{v}=H_{z}^{p}
\end{array}
$$

where the superscript $v$ denotes vacuum, and $p$ the plasma region. Across the current sheet at $r=b$, the tangential components of the electric field are continuous and those of the magnetic field are discontinuous, according to Ampere's law. Therefore, we can write

$$
\begin{gathered}
E_{\phi}^{1}=E_{\phi}^{2}, \quad E_{z}^{1}=E_{z}^{2} \\
H_{z}^{2}-H_{z}^{1}=J_{\phi} \\
H_{\phi}^{1}-H_{\phi}^{2}=J_{z}
\end{gathered}
$$

where regions 1 and 2 are shown in Fig. 2.

\section{A. Current Distribution on the Antennas}

The current distribution characterizing the antennas shown in Fig. 1 is calculated in this section. Due to the uniformity of the geometry in the $\phi$ and $z$ directions, the spatial variation of the current distribution of the antennas can be represented by an infinite set of current sheets with the complex exponential form $e^{i m \phi+i k_{z} z}$. The $m-k_{z}$ spectrum of the antennas, that is, the Fourier coefficients of the current density, can be applied directly since the current distribution is contained in a cylindrical surface of constant radius.

Assuming the current density of the antennas to be solenoidal $(\nabla \cdot \boldsymbol{J}=0)$, we need to determine only the azimuthal current density since the axial current density can be calculated from the $m-k_{z}$ transform of the continuity equation. That is

$$
\nearrow_{z}\left(m, k_{z}\right)=-\frac{m}{b k_{z}} J_{\phi}\left(m, k_{z}\right)
$$

The above low-frequency approximation of a stationary current distribution for the antennas $(\nabla \cdot \boldsymbol{J}=0)$ implies that the electrostatic fields due to the free charge on the antennas are effectively shielded from the plasma and can be neglected.

1) Fractional Helix Coil: The fractional or partial-turn helical antenna is diagrammed in Fig. 1. The fractional helix in the limit of straight helical windings models the Nagoya typeIII coil. The current distribution is expressed in terms of delta and heaviside functions

$$
\begin{aligned}
J_{\phi}(r, \phi, z)= & \frac{I_{0}}{2} \delta_{r}(b)\left\{\delta\left(z-\frac{L}{2}\right)\right. \\
& \cdot\left[u_{\phi}(\theta, \pi+\theta)-u_{\phi}(-\pi+\theta, \theta)\right]+\delta\left(z+\frac{L}{2}\right) \\
& \left.\cdot\left[u_{\phi}(-\pi-\theta,-\theta)-u_{\phi}(-\theta, \pi-\theta)\right]\right\} \\
& +I_{0} \delta_{r}(b)\left[\delta\left(z-\frac{L}{2 \theta} \phi\right)\right. \\
& \left.-\delta\left(z-\frac{L}{2 \theta} \phi+\frac{\pi L}{2 \theta}\right)\right]
\end{aligned}
$$

where

$L=$ length of the coil,

$\theta=\pi l=\left(180^{\circ}\right)$ (number of helical turns). 
The delta function is used to constrain the flow of the current to radius $r=b$. The heaviside function $u_{z}[ \pm(w / 2)]$ is defined as

$$
\begin{aligned}
u_{z}\left(-\frac{w}{2},+\frac{w}{2}\right) & =u_{z}\left( \pm \frac{w}{2}\right) \\
& = \begin{cases}1 & \text { for }-\frac{w}{2}<z<+\frac{w}{2} \\
0 & \text { otherwise. }\end{cases}
\end{aligned}
$$

The $m-k_{z}$ transform is determined from (2)

$$
\begin{aligned}
J_{\phi}\left(r, m, k_{z}\right)= & \frac{2 I_{0}}{\pi} \delta_{r}(b) \theta\left[\frac{\sin \left(\frac{k_{z} L}{2}+m \theta\right)}{\frac{k_{z} L}{2}+m \theta}\right]+\frac{i I_{0}}{m \pi} \delta_{r}(b) \\
& \cdot\left[e^{i\left(k_{z} L / 2\right)} e^{i m \theta}-e^{-i\left(k_{z} L / 2\right)} e^{-i m \theta}\right]
\end{aligned}
$$

The fractional helix reduces to the Nagoya type-III case when $\theta=0$.

\section{Helicon Source Modeling Results}

\section{A. Modifications of the "ANTENA" Code}

The "ANTENA" code, written by McVey [11], [12], was programmed in FORTRAN language on the CRAY CTSS computer system, to study ICRF (ion cyclotron range of frequencies) heating of magnetic fusion plasmas. We have ported the code from the CRAY facilities to our UNIX-based workstations using the graphics package PIPlot.

We decreased the cylindrical shell width and checked convergence (typically 800 radial points) for 2.5 - and $5.0-\mathrm{cm}$ radii stratified plasma density and temperature profiles. This allows us to study the propagation of helicon waves in the frequency range, $f \sim 1-30 \mathrm{MHz}$, which exhibit short wavelengths in the radial profile $\left(\lambda_{\perp} \geq 1 \mathrm{~mm}\right)$. A typical run with 800 strata (radial points) describing the stratified plasma density and temperature profiles and assuming only one azimuthal mode requires about five minutes of run time on an IBM RS/6000 model 370 workstation.

Helical coils, such as the fractional and integral helix shown in Fig. 1 have been analyzed and added to the code to examine different types of helicon sources. The Stix coil [13], [16], which consists of an array of full-turn loops separated by a distance of $\lambda / 2$ and having alternative current flow directions, has been also modeled. Connecting two loops by a straight horizontal wire provides a more accurate description of the Stix coil. The Stix coil with the horizontal wire connection can also excite other modes in addition to the $m=0$ mode. We have found that the contribution to the radiation resistance of the $m=+1$ and higher order modes is negligible compared to the $m=0$ mode, and thus we will focus on the model having only the two full-turn loops for the description of the Stix coil.

\section{B. Antenna Coupling}

The antennas diagrammed in Fig. 1 were modeled to primarily excite $m=+1$ and $m=0$ azimuthal modes for plasma production. The Nagoya type-III [17] and the helical coils primarily excite the $m=+1$, and the Stix coil, due to its azimuthal uniformity, primarily excites the $m=0$ mode.
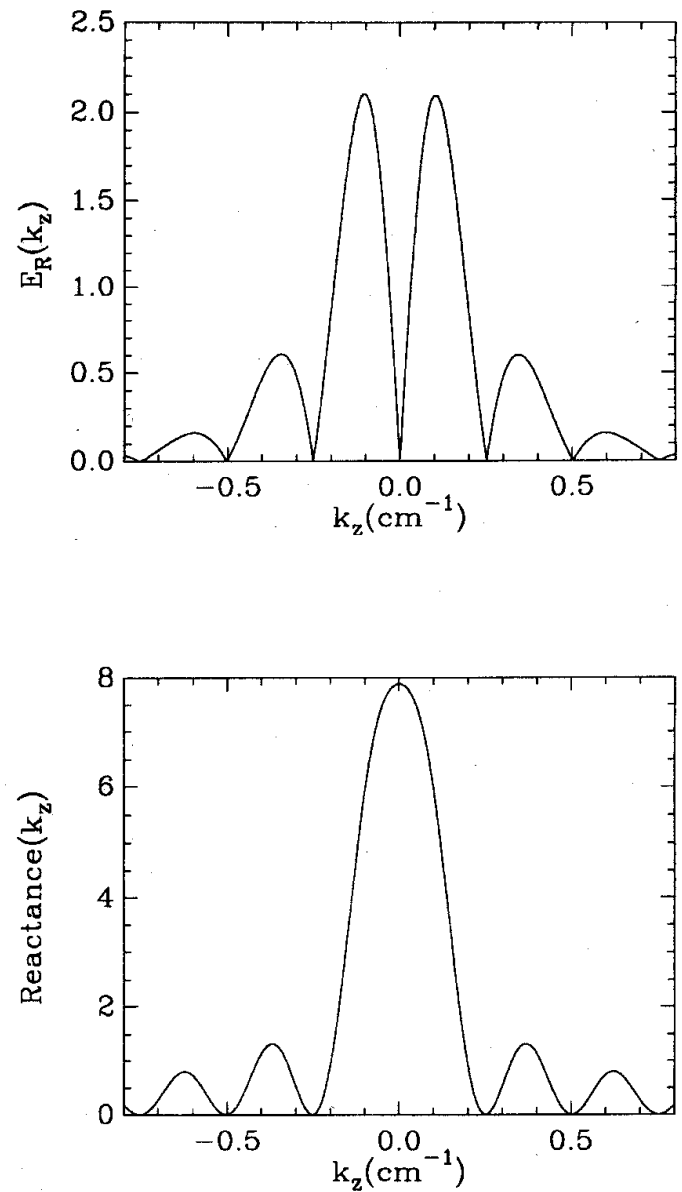

Fig. 3. Vacuum antenna spectra of Nagoya type-III antenna for an $m=+1$ azimuthal mode.

The vacuum spectra of $E_{\text {right }}=\left|E_{x}-i E_{y}\right| / 2$, for a Nagoya type-III antenna of length $L=25 \mathrm{~cm}$, and radius $r=5.5$ $\mathrm{cm}$, and considering the dominant $m=+1$ azimuthal mode at $r=1 \mathrm{~cm}$ is shown in Fig. 3. Looking at the $E_{R}\left(k_{z}\right)$-spectrum for this antenna, we see that it couples best to vacuum near $k_{z}\left(\mathrm{~cm}^{-1}\right) \approx 0.12(\approx \pi / L), 0.36(\approx 3 \pi / L), 0.6(\approx 5 \pi / L)$, etc., corresponding to $\lambda_{z}(\mathrm{~cm}) \approx 52.35(\approx 2 L), 17.45(\approx$ $2 L / 3), 10.47(\approx 2 L / 5)$, etc. Fig. 4 shows the vacuum $E_{R}\left(k_{z}\right)$ spectrum of a fractional helix antenna with the same selected dimensions and azimuthal mode number the same as that of the Nagoya type-III antenna $(m=+1)$. The spectrum of this antenna is not symmetric in $k_{z}$ and it couples directionally to the helicon mode for $k_{z}\left(\mathrm{~cm}^{-1}\right) \approx 0.18,0.48,0.72$, etc., corresponding to $\lambda_{z}(\mathrm{~cm}) \approx 34.9,13.08,8.7$, etc.

Both antennas excite a number of odd $m$ modes $(m=$ $\pm 3, \pm 5, \pm 7, \pm 9, \cdots)$ in vacuum with amplitudes that decrease as $1 / \mathrm{m}^{2}$. In a plasma, as will be shown later (Fig. 9) for the Nagoya type-III and the fractional helix antenna for the plasma parameters shown, the $E_{z}\left(k_{z}\right)$-spectrum narrows significantly because of the excitation of the helicon eigenmode at $k_{z}\left(\mathrm{~cm}^{-1}\right) \approx 0.125$. The $E_{z}\left(k_{z}\right)$-spectrum for both antennas peaks at about the same $k_{z}$ with the fractional helix having a higher peak value of field intensity $(\times 1.15)$ at radial position $r=0.75 \mathrm{~cm}$ for this $m=+1$ azimuthal mode. The helix, in 


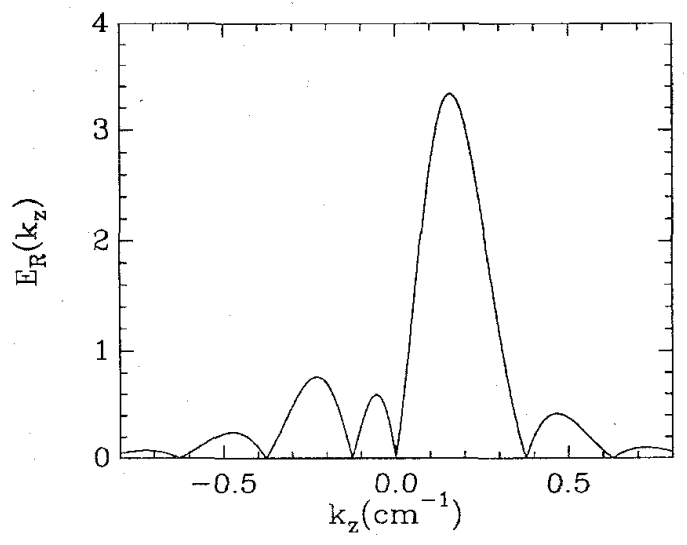

TABLE I

IMPEDANCE AND QUALITY FACTOR $\left(Q_{p}\right)$ FOR VARIOUS ANTENNAS IN A PaRabolic Plasma DenstTy Profrle $n_{e}(r)=n_{e}\left(1-(r / a)^{2}\right)$ AND UnIform TeMPERATURe PROFLL $\left(B_{0}=700 \mathrm{G}, n_{e}=2 \times 10^{13} \mathrm{~cm}^{-3}\right.$, $\left.T_{e 0 \text {-fast }}=40 \mathrm{eV}, T_{e 0 \text {-slow }}=3 \mathrm{eV}, f=7 \mathrm{MHz}\right)$

\begin{tabular}{cccc}
\hline Antenna & Resistance & Reactance & $Q_{p}=\omega L / R$ \\
\hline & $(\Omega)$ & $(\Omega)$ & \\
\hline \hline Nagoya type III & 1.36 & 7.70 & 5.66 \\
Fractional helix & 2.54 & 7.87 & 3.10 \\
Stix & 0.24 & 2.12 & 8.83 \\
\hline
\end{tabular}

TABLE II

IMPEDANCE AND QUALITY FACTÓR $\left(Q_{p}\right)$ FOR VARIOUS ANTENNAS IN A PLASMA DENSITY PROFIL OF THE FORM $n_{e}(r)=n_{e 0}\left(1-(r / a)^{2}\right)^{3}$ AND UNIFORM TeMPerature Profile $\left(B_{0}=700 \mathrm{G}, n_{e 0}=2 \times 10^{13} \mathrm{~cm}^{-3}\right.$ $\left.T_{e 0 \text {-fast }}=40 \mathrm{eV}, T_{\text {e0-slow }}=3 \mathrm{eV}, f=7 \mathrm{MHz}\right)$

\begin{tabular}{cccc}
\hline Antenna & Resistance & Reactance & $Q_{p}=\omega L / R$ \\
\hline & $(\Omega)$ & $(\Omega)$ & \\
\hline Nagoya type III & 0.72 & 10.10 & 14.02 \\
Fractional helix & 2.11 & 10.20 & 4.83 \\
Stix & 0.14 & 2.17 & 15.17 \\
\hline
\end{tabular}

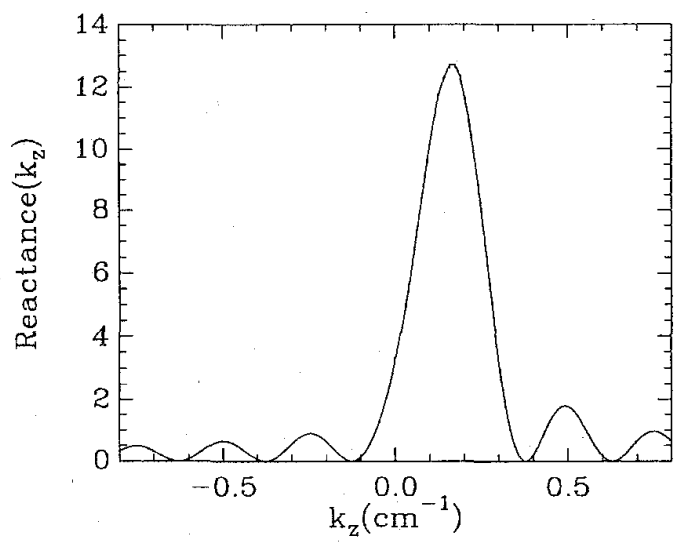

TABLE III

IMPEDANCE AND QuALITY FACTOR $\left(Q_{p}\right)$ FOR VARIOUS ANTENNAS IN A PLASMA DENSTTY PROFIL OF THE FORM $n_{e}(r)=n_{e 0}\left(1-(r / a)^{2}\right)^{5}$ AND UnfForm Temperature Profule $\left(B_{0}=700 \mathrm{G}, n_{e 0}=2 \times 10^{13} \mathrm{~cm}^{-3}\right.$ $T_{\text {e0-fast }}=40 \mathrm{eV}, T_{\text {e0-slow }}=3 \mathrm{eV}, f=7 \mathrm{MHz}$ )

\begin{tabular}{cccc}
\hline Antenna & Resistance & Reactance & $Q_{p}=\omega L / R$ \\
\hline & $(\Omega)$ & $(\Omega)$ & \\
\hline Nagoya type III & 0.35 & 11.30 & 32.28 \\
Fractional helix & 1.73 & 11.40 & 6.59 \\
Stix & 0.10 & 2.23 & 21.44 \\
\hline
\end{tabular}

Fig. 4. Vacuum antenna spectra of fractional helix antenna for an $m=+1$ azimuthal mode.

contrast to the Nagoya type-III, is strongly asymmetric in its $E_{z}\left(k_{z}\right)$ spectra in plasma.

The "ANTENA" code has the capability of computing the radiation resistance and reactance of the inductive antennas shown in Fig. 1. The power transfered to the plasma can be written as

$$
P_{c}=-\frac{1}{2} \int E \cdot J^{*} d V
$$

The impedance of the antenna is then written as

$$
\begin{aligned}
Z & =\frac{2 P_{c}(w)}{|I|^{2}} \\
& =R_{A}+i X_{A}
\end{aligned}
$$

where

$R_{A}=R_{r}+R_{L}=$ antenna resistance

$R_{r}=$ radiation resistance of the antenna,

$R_{L}=$ skin loss resistance of the antenna,

$X_{A}=$ antenna reactance.

The radiation resistance and the quality factor $Q$ are important parameters in determining the efficiency of coupling power to the plasma. Radiated power contributes to the real part of the antenna impedance whereas power stored in the near field is represented by the reactive part of the impedance.

The overall quality factor, $Q$, can be determined from the plasma $Q_{p}=\omega L / R$, calculated from "ANTENA" and results are shown in Tables I-III, and the external matching network $Q_{c}$. That is, $1 / Q=1 / Q_{p}+1 / Q_{c}$.

The radiation resistance of the fractional helix, the Nagoya type-III antenna, and the Stix coil for three types of plasma density profiles was computed by integrating over all the azimuthal mode numbers $m$. Tables I-III summarize these results when both collisional and Landau damping processes are included. The antenna is centered at $z=0 \mathrm{~cm}$, with length $L=25 \mathrm{~cm}$ and radius $r=5.5 \mathrm{~cm}$. The helical antenna has a much higher value of radiation resistance and lower quality factor than the Nagoya type-III for all three plasma profiles modeled. Therefore, this antenna is more easily matched to an external generator and efficiently couples power to the plasma. The higher radiation resistance of the helical antenna also allows efficient power coupling to the plasma with minimal skin effect losses on the coil structure. The impedance of the Stix coil $(m=0)$ was also computed. The Stix coil excites a low-amplitude $E_{z}\left(k_{z}\right)$-spectrum compared to the other antennas as we will discuss later. It has the smallest radiation resistance and also a large reactance which results in a very large value of the quality factor $Q$. Therefore, it is it more difficult to match the input power from the RF source to the antenna. 


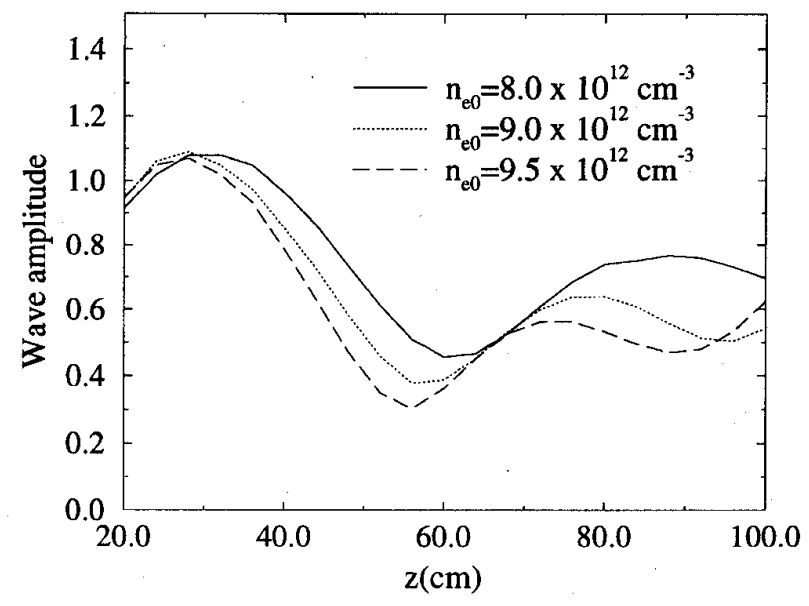

Fig. 5. Spatial variation of damped waves with increasing plasma density at fixed frequency $f=6 \mathrm{MHz}$ and magnetic field $B_{0}=800 \mathrm{G}$ for a Nagoya type-III antenna.

Tables I-III show that the fractional helix is superior for coupling power to the plasma. The lower $Q$ for the helical sources indicates that lower inductive stored field energy is required for a given time-averaged power dissipated in the plasma electrons. The higher $Q$ for the Stix coil and Nagoya type-III implies that larger field strengths are produced, which lead to inductive stored energy which does not heat the plasma. As the assumed plasma density and its gradient at the edge are reduced, it increases the reactive stored energy in the outer regions, and therefore decreases the absorption there. The fractional helix is well suited as a source for all cases since the radiation resistance is larger and its quality factor, $Q$, is lower than the other two antennas studied. As a result, the fractional helix provides efficient heating of the plasma with more efficient use of the $k_{z}$ antenna spectrum which couples well to core electrons.

\section{Helicon Source Modeling Results}

The "ANTENA" code was used to model cases corresponding to selected experimental data on helicon sources [4]. The approximate dispersion relation for the $m=+1$ helicon mode can be written as [8]

$$
\frac{\omega}{k_{z}}=\frac{3.83 B_{0}}{a n_{e} e \mu_{0}} .
$$

The axial wavelength of this mode is then obtained

$$
\lambda_{z}=\frac{3.83 B_{0}}{a n_{e} e \mu_{0} f} \text {. }
$$

That is, for a given frequency $f$, and magnetic field $B_{0}$, the helicon wavelength will vary roughly as $1 / n_{e}$. Fig. 5 shows the variation of the spatially damped $B_{z}$ wave amplitude with increasing electron density $n_{e}$, assuming a parabolic plasma density, $n_{e}(r)$, and plasma temperature $T_{e}(r)$. The length of the Nagoya type-III antenna is $L=25 \mathrm{~cm}$ and is centered at $z=0 \mathrm{~cm}$. We observe that the axial wavelength decreases with increasing density, as expected, and the wavelength is about $50 \mathrm{~cm}$, which is twice the antenna length. Fig. 6 shows the axial variation of the amplitude of the magnetic field

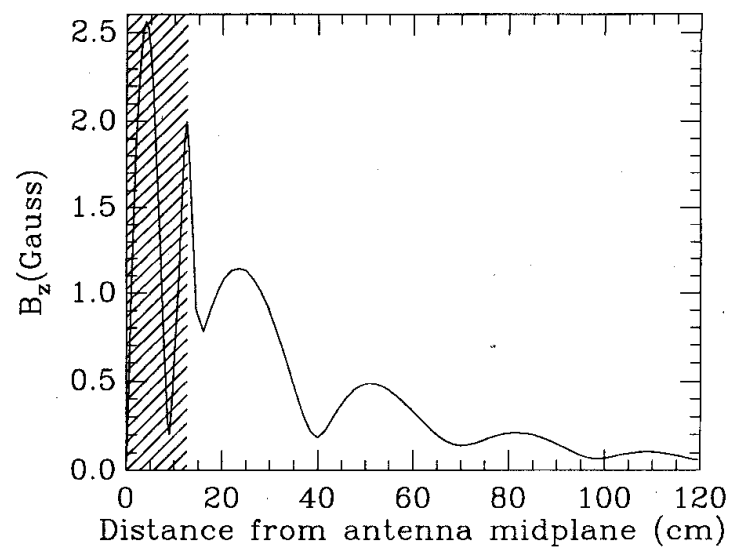

Fig. 6. Axial variation of the amplitude of the magnetic field at fixed frequency $f=27 \mathrm{MHz}$ and magnetic field $B_{0}=800 \mathrm{G}$ for the Nagoya type-III antenna of length $L=25 \mathrm{~cm}$.

for a frequency $f=27 \mathrm{MHz}$, an on-axis plasma density $n_{e 0}=2 \times 10^{13} \mathrm{~cm}^{-3}$, and a magnetic field $B_{0}=800 \mathrm{G}$ for a Nagoya type-III antenna. The antenna is positioned within the shaded region. The amplitude of the wave $B_{z}$ magnetic field peaks under the antenna and then manifests a decaying wave as one moves away from the antenna, in agreement with experimental observations [18].

We have studied plasma density and temperature profile effects on power absorption of a small fraction $\left(n_{f e} / n_{e} \approx 5 \%\right)$ of fast electrons $\left(T_{e} \approx 40 \mathrm{eV}\right)$ and bulk $\left(T_{e} \approx 3 \mathrm{eV}\right)$ electron distributions in the model. These fast electrons, which are necessary to ionize neutrals and sustain the plasma discharge against recombination and other losses, have been observed in some experiments [9], [5], [10]. Figs. 7 and 8 illustrate the radial power absorbed by the bulk and fast electrons for the three coils we have modeled. The solid and dotted curves correspond, respectively, to the slow and fast electrons.

In recent helicon experiments [4], the electron temperature was measured to be approximately uniform across the plasma. A plasma density profile with a peak on axis and a small but finite value at the edge (see solid curve in Fig. 10), along with a uniform temperature profile is used in the model corresponding to the experimental plasma profiles measured by Komori et al. [4]. Figs. 7-9 and 11 correspond to simulations for these assumed profiles. By using this uniform plasma temperature profile we provide a collisional heating model which smoothly decreases as one moves to the edge of the plasma, due to the strong temperature dependence of the collision frequency $\nu(r) \sim T_{e}^{-3 / 2}$ in the Krook collisional model.

The radial power absorbed by the plasma $\left[P\left(\mathrm{~W} / \mathrm{cm}^{2}\right)\right]$ is the total power absorbed in an infinitely long cylindrical shell of radius $r$. The total power absorbed by the plasma can be computed by integrating over the cross section. That is, $P_{\text {total }}=2 \pi \int_{0}^{r=a} P\left(\mathrm{~W} / \mathrm{cm}^{2}\right) r d r$. This total coupled power is kept constant $\left(P_{\mathrm{RF}}=700 \mathrm{~W}\right)$ in our model by varying the current on the antennas.

The fractional helix that was first used by Shoji [19] was modeled and either the $m=+1$ or $m=-1$ mode could be excited corresponding to coil rotation. Fig. 7(b) shows that 


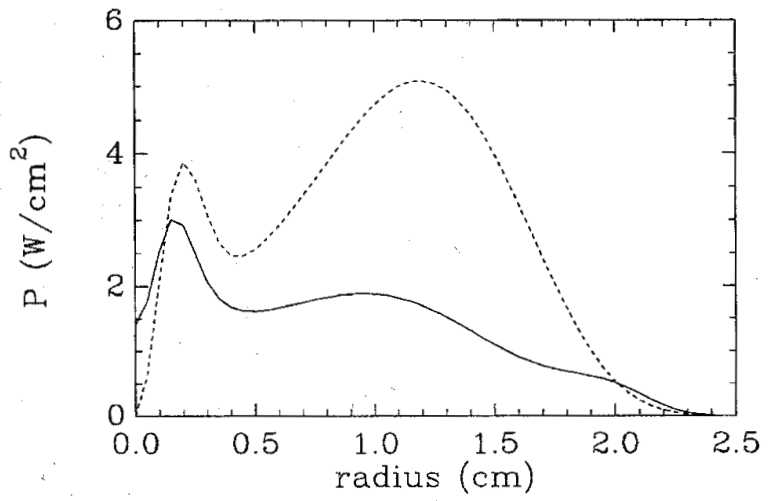

(a)

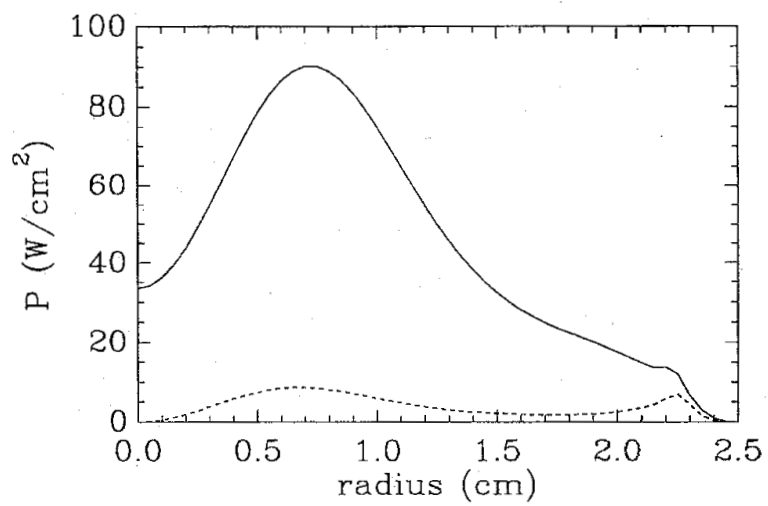

(b)

Fig. 7. Radial power absorbed by the bulk (solid curve) and fast (dotted curve) electrons for a fractional helix antenna, due to Landau and collisional damping of the helicon waves for the $m=+1$ mode and frequency $f=$ $7 \mathrm{MHz}$. (a) Low density $\left(n_{e 0}=1 \times 10^{11} \mathrm{~cm}^{-3}, B_{0}=250 \mathrm{G}\right)$. (b) High density $\left(n_{e 0}=2 \times 10^{13} \mathrm{~cm}^{-3}, B_{0}=700 \mathrm{G}\right)$.

this antenna couples to a broad $(m=+1)$ plasma absorption profile at high densities $\left(n_{e 0} \approx 2 \times 10^{13} \mathrm{~cm}^{-3}\right)$ in agreement with the experiments done by Komori [4].

The Stix coil [13], [16] has a heating profile and an electric field $E_{z}\left(k_{z}\right)$ spectrum as shown in Figs. 8(b) and 9(c). The model shows that the $m=0$ azimuthal mode fields provide substantial core heating. It also suggests that the power absorbed by the plasma for all three antennas used is, in part, due to Landau damping of the wave which heats the fast electrons. This is illustrated in Fig. 9(a)-(c), where the $E_{z}\left(k_{z}\right)$-spectrum at the radial position $r=0.75 \mathrm{~cm}$ peaks at about $\omega / k_{z} v_{t h} \approx \sqrt{2}$, where the Landau damping rate is maximized. These fast electrons absorb the RF power preferentially at the edge for high on-axis densities $\left(n_{e 0} \approx\right.$ $\left.2 \times 10^{13} \mathrm{~cm}^{-3}\right)$.

1) Collisional Effects on the Power Absorbed Due to $\nu\left(n_{e}, T_{e}\right)$ Profile Effects: The competing effects of Landau and collisional damping of the helicon waves which heat the electrons have been investigated in the density range of $n_{e} \approx 5 \times 10^{10}-5 \times 10^{13} \mathrm{~cm}^{-3}$. A radial density and temperature-dependent collision frequency, $\nu(r) \sim n_{e} / T_{e}^{3 / 2}$, were added to the "ANTENA" code.

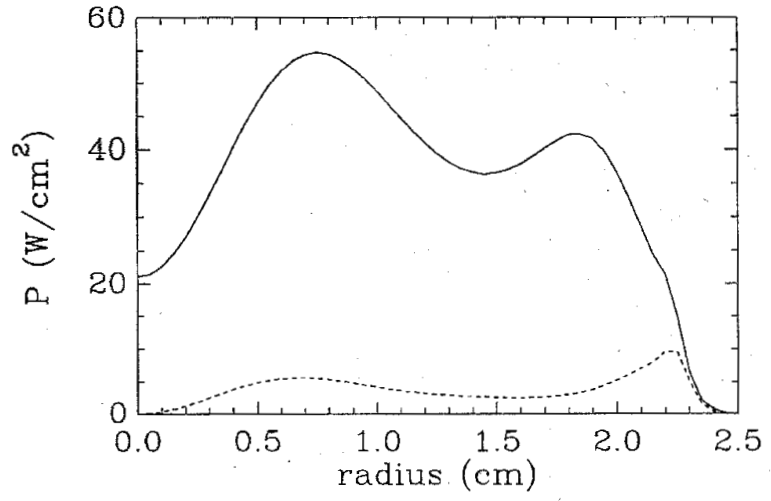

(a)

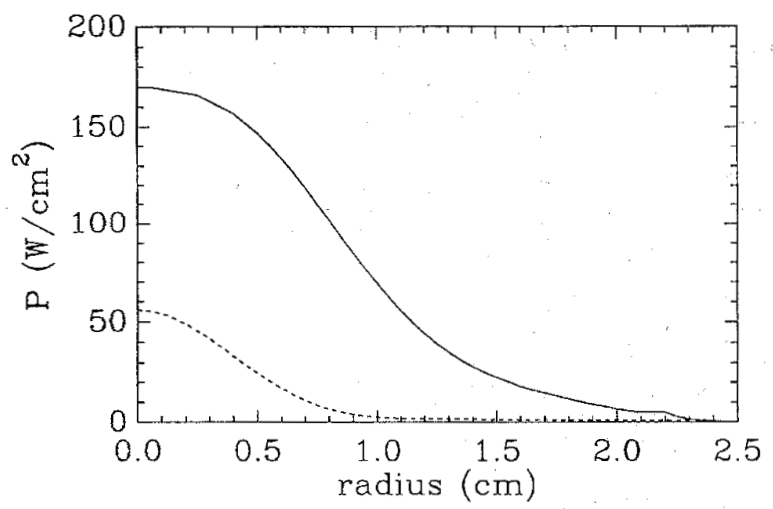

(b)

Fig. 8. Radial power absorbed by the bulk slow (solid curve) and fast (dotted curve) electrons due to Landau and collisional damping of the helicon waves for the $m=+1$ mode and $B_{0}=700 \mathrm{G}, n_{e 0}=2 \times 10^{13} \mathrm{~cm}^{-3}, f=7$ $\mathrm{MHz}$. (a) Nagoya type-III antenna. (b) Stix coil antenna.

We have modeled low- and high-plasma density regimes as shown in Fig. 7. We fixed the frequency at $f=7 \mathrm{MHz}$ and varied the magnetic field $B_{0}$ to keep the ratio $B_{0} / n_{e}$, and therefore the helicon axial wavelength $\lambda_{\|}$[see (24)] constant. This axial wavelength is in the range between 10 and $60 \mathrm{~cm}$ for the parameters used in our model. The fractional helix antenna with dimensions as described earlier was used to excite the $m=+1$ mode. Fig. 7 illustrates that both Landau and collisional damping processes occur in this density range. At low densities, as illustrated in Fig. 7(a), most of the RF power is absorbed by the small fraction of fast electrons via Landau damping of the helicon wave. As the plasma density increases, the absorption by the bulk slow electrons due to collisional damping becomes dominant and the collisional damping rate becomes larger than that due to the Landau process. At high densities $\left(n_{e} \geq 10^{13} \mathrm{~cm}^{-3}\right)$, collisional damping is the dominant heating mechanism in these $3-4 \mathrm{eV}$ plasmas, but a small Landau absorption on fast electrons exists.

We have modeled various plasma density profiles according to the two-parameter dependent curve $n_{e}(r)=n_{e 0}(1-$ $\left.(r / a)^{s}\right)^{t}$, to examine their effect on the power absorption. Various pairs of the parameters $(s, t)$, assuming a uniform temperature profile, are shown in Fig. 10(a). The model which includes both Landau and collisional heating mechanisms 


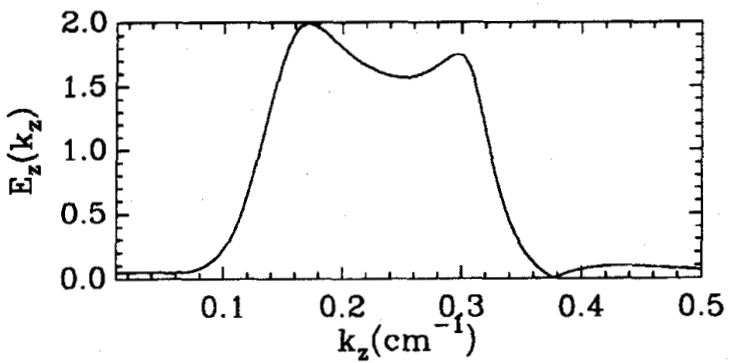

(a)

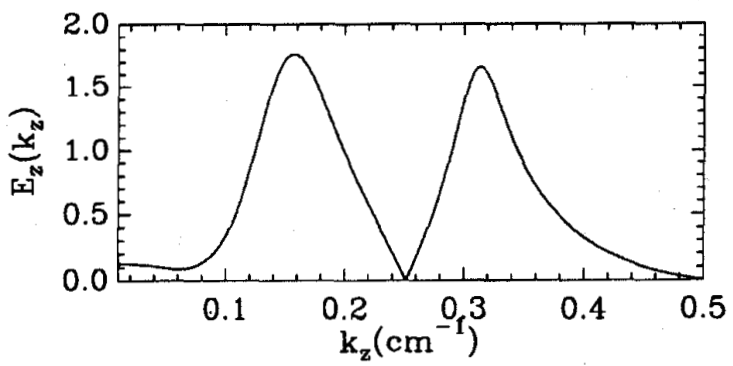

(b)

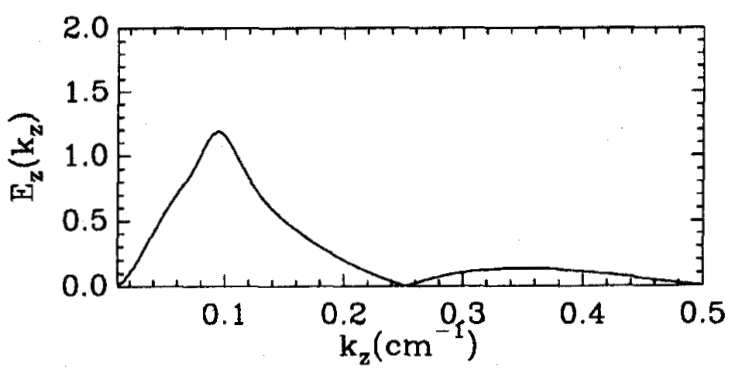

(c)

Fig. 9. $E_{z}\left(k_{z}\right)$-spectrum in plasma for various antennas including Landau and collisional damping of helicon waves at radial position $r=0.75 \mathrm{~cm} . B_{0}=700 \mathrm{G}, n_{e 0}=2 \times 10^{13} \mathrm{~cm}^{-3}, f=7 \mathrm{MHz}$. (a) Fractional helix ( $m=+1$ mode). (b) Nagoya type-III ( $m=+1$ mode). (c) Stix coil ( $m=0$ mode).

shows that the power absorbed is quite sensitive to these assumed profiles. The density profiles with a steep density gradient near the edge, such as the ones with parameters $(s=4, t=1),(s=2, t=1)$, lead to substantial edge heating, as is shown in Fig. 10(b). The high fields at the edge that are responsible for the heating of the plasma electrons via Landau damping of the wave buildup through reflections that occur when the wave sees this steep density gradient as it propagates radially inwards. Smoother profiles, such as $(s=2, t=2),(s=2, t=3),(s=2, t=4)$, allow the waves to penetrate to the center, and therefore core heating becomes dominant.

The electron heating profile, as well as the antenna performance, is also affected by edge density profile effects. A steep radial density gradient at the edge provides a higher radiation resistance (see Tables I-III), along with significant edge heating.

The efficiency of three antennas (Nagoya type-III, helical, and Stix) has also been studied for the given plasma parame-

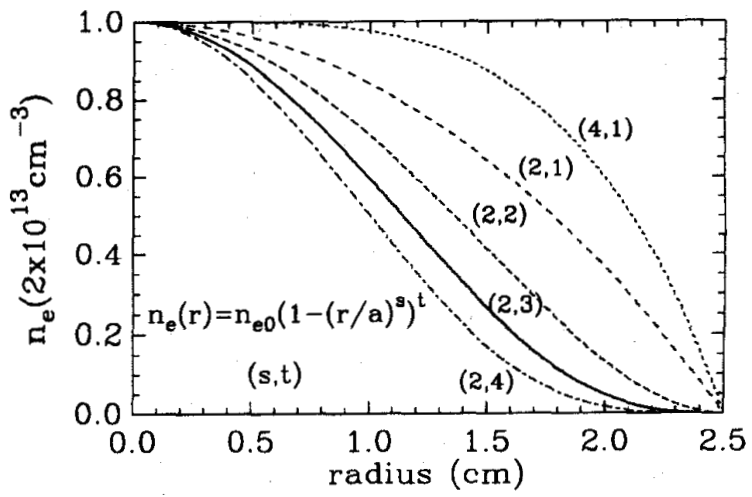

(a)

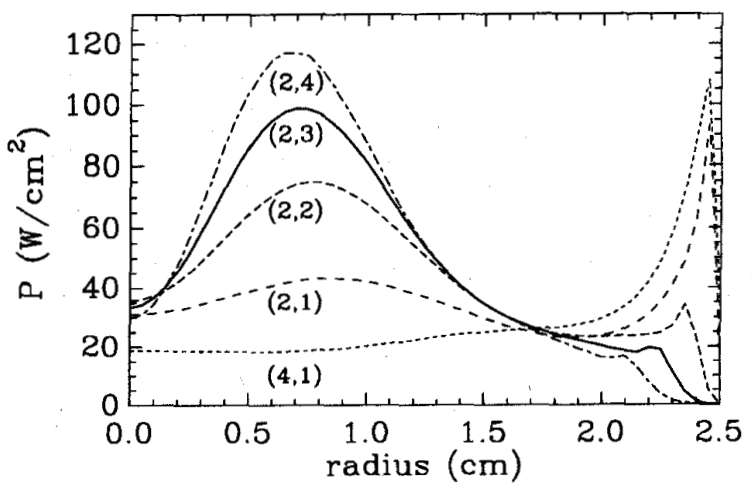

(b)

Fig. 10. (a) Plasma density radial profiles modeled for a fractional helix antenna. (b) Radial power absorption profiles for the density profiles in (a) and assuming uniform temperature profile for the $m=+1$ azimuthal mode, due to Landau and collisional damping. $B_{0}=700 \mathrm{G}, n_{e 0}=2 \times 10^{13} \mathrm{~cm}^{-3}, f=7$ $\mathrm{MHz}$.

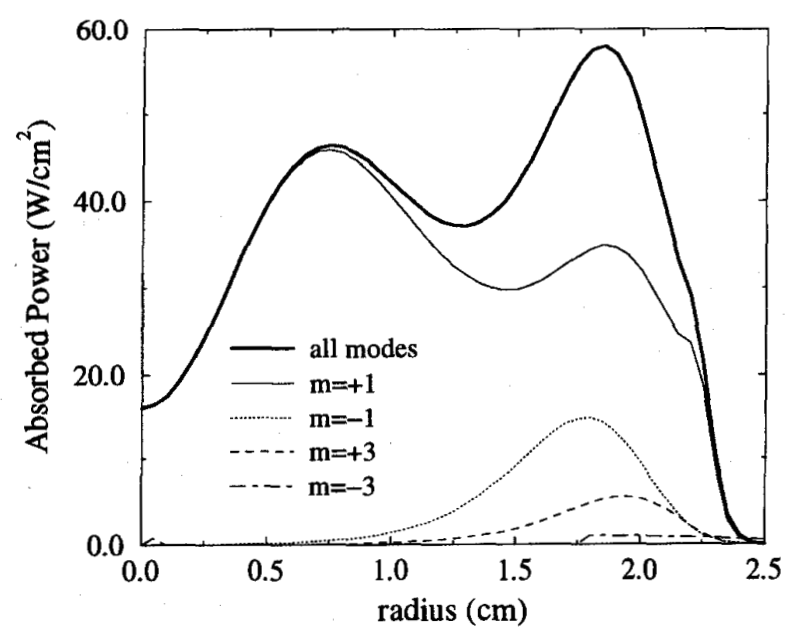

Fig. 11. Total radial power absorbed by the plasma for a Nagoya type-III antenna due to Landau and collisional damping of the helicon waves. $B_{0}=700 \mathrm{G}, n_{e 0}=2 \times 10^{13} \mathrm{~cm}^{-3}, f=7 \mathrm{MHz}$.

ters. Examining Figs. 7, 8, and 9, we observe that the Nagoya type-III antenna and the partial-turn helix excite similar high 
$E_{z}\left(k_{z}\right)$-spectra for the $m=+1$ azimuthal mode and have similar radial profiles for the absorbed power, with the helical antenna providing a higher amplitude of the power absorbed by the electrons near the axis due to its higher radiation resistance. The Stix coil which excites a relatively lower amplitude $E_{z}\left(k_{z}\right)$-spectrum for the dominant $m=0$ mode, provides a narrow but substantial radial heating profile.

We also show in Fig. 11 that the Nagoya type-III antenna excites a number of azimuthal $m$ modes. These higher order modes heat the plasma primarily near the edge. The fractional helix antenna excites only the $m=+1$ mode and reduces the edge heating process.

\section{SUMMARY AND CONCLUSIONS}

The computer code "ANTENA" was used to study and model helicon sources. The Nagoya type-III, the partial-turn helix, and the Stix coil antenna were modeled to examine the plasma absorption profile in the lower hybrid frequency range. The Stix coil, which excites the $m=0$ mode, heats the plasma primarily in the center. This RF coil structure should be considered together with the helix and the Nagoya type-III helicon plasma sources. The partial-turn helix and the Nagoya type-III coils excite the $m=+1$ mode, the dominant mode for this frequency range $(f=1-30 \mathrm{MHz})$. We have included a small fraction $\left(n_{f e} / n_{e} \approx 5 \%\right)$ of fast electrons ( $T_{e \text {-fast }} \approx$ $40 \mathrm{eV}$ ) in the model and have shown that these fast electrons absorb most of the RF power via Landau damping at low densities $\left(n_{e} \leq 10^{12} \mathrm{~cm}^{-3}\right)$.

The partial-turn helix is found to be more efficient in coupling the power to the plasma than the Nagoya type-III for our assumed plasma profiles. This is due to its negligible excitation of higher order $(m \geq 3)$ modes which raise the reactance of the source and increase the edge heating effects, higher radiation resistance of the partial-turn helix antenna, and lower quality factor, $Q_{p}$, of this plasma source. The helix also provides an improved directionality (more launched wave power in one direction from the source) of the $k_{z^{-}}$ spectrum.

We have added a radially varying collision frequency model $\left[\nu\left(n_{e}(r), T_{\epsilon}(r)\right)\right]$ to the code to study the effects and importance of the collisional and Landau damping heating mechanisms. The Krook collisional model shows that collisions play an important role in plasma production, and is the dominant electron heating mechanism at high densities $\left(n_{e} \geq\right.$ $\left.10^{13} \mathrm{~cm}^{-3}\right)$. We find that electron heating profiles are very dependent on the plasma and collisional profiles. Plasma density profiles with a steep gradient at the edge lead to substantial edge heating. Smoother profiles allow the waves to penetrate and dissipate their energy in the center, which is comparable to experimental observations. In future work, we plan to examine the influence of a small fraction of fast electron beams which have been observed experimentally on helicon wave power absorption.

\section{ACKNOWLEDGMENT}

The authors would like to thank B. McVey for providing a copy of his computer code "ANTENA," along with his helpful comments and suggestions. We are also grateful to M. Bettenhausen for many valuable discussions.

\section{REFERENCES}

[1] D. M. Manos and D. L. Flamm, Plasma Etching. An Introduction. New York: Academic, 1989.

[2] M. A. Lieberman and R. A. Gottscho, "Design of high density plasma sources for materials processing," University of California, Berkeley, Rep. UCB/ERL M93/3, 1993.

[3] R. W. Boswell, "Very efficient plasma generation by whistler waves near the lower hybrid frequency," Plasma Phys. Controlled Fusion, vol. 26(10), pp. 1147-1162, 1984.

[4] A. Komori, T. Shoji, K. Miyamoto, J. Kawai, and K. Kawai, "Helicon waves and efficient plasma production," Phys. Fluids B, vol. 3(1), pp. 893-898, 1991.

[5] F. F. Chen and C. D. Decker, "Electron acceleration in helicon sources," Plasma Phys. Controlled Fusion, vol. 34(4), pp. 635-640, 1992.

[6] T. Shoji, Y. Sakawa, S. Nakazawa, K. Kadota, and T. Sato, "Plasma production by helicon waves," Plasma Sources Sci. Technol., vol. 2(11), pp. 5-10, 1993.

[7] R. W. Boswell, "Plasma production using a standing helicon wave," Phys. Lett., vol. 33A(7), pp. 457-458, 1970.

[8] F. F. Chen, "Plasma ionization by helicon waves," Plasma Phys. Controlled Fusion, vol. 33(11), pp. 339-364, 1991.

[9] P. K. Loewenhardt, B. D. Blackwell, R. W. Boswell, G. D. Conway, and S. M. Hamberger, "Plasma production in a toroidal heliac by helicon waves," Phys. Rev. Lett., vol. 67(20), pp. 2792-2794, 1991.

[10] P. K. Loewenhardt, B. D. Blackwell, and S. M. Hamberger, "Production of fast electrons in a prototype heliac by helicon waves," Phys. Plasmas, vol. 1(4), pp. 875-880, 1994.

[11] B. D. McVey, "Antena user guide," Plasma Fusion Ctr., MIT, Rep. PFC/RR-84-13, 1984.

[12] "ICRF antenna coupling theory for a cylindrically stratified plasma," Plasma Fusion Ctr., MIT, Rep. PFC/RR-84-12, 1984.

[13] T. H. Stix, Waves in Plasmas. New York: Amer. Inst. Phys., 1992.

[14] P. L. Bhatnagar, E. P. Gross, and M. Krook, "A model for collision processes in gases. X. Small amplitude processes in charged and neutral one-component systems," Phys. Rev., vol. 94(3), pp. 511-522, 1954.

[15] B. D. Fried and S. D. Conte, The Plasma Dispersion Function. New York: Academic, 1961.

[16] J. C. Hosea and R. M. Sinclair, "Ion cyclotron wave generation in the model C stellarator," Phys. Fluids, vol. 13(3), pp. 701-711, 1970.

[17] K. Watari et al., "Radio-frequency plugging of a high density plasma," Phys. Fluids, vol. 21(11), pp. 2076-2081, 1978.

[18] I. Sudit, private communication, 1995.

[19] T. Shoji, TPPJ Annu. Rev., Nagoya Univ., Rep. 63, 1986.

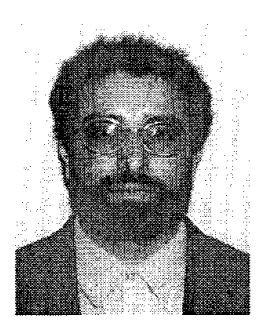

Yiannis Mouzouris was born in Limassol, Cyprus, on May 7, 1969. He received the B.S. and M.S. degrees in electrical engineering from the University of Wisconsin, Madison, in 1992 and 1994, respectively. He is presently pursuing the Ph.D. degree at the University of Wisconsin-Madison.

His research interests include modeling of highdensity plasma sources for materials processing and ICRF wave heating in tokamak plasmas.

John E. Scharer (M'90) received the B.S., M.S., and Ph.D. degrees in electrical engineering from the University of California, Berkeley, in plasma physics.

$\mathrm{He}$ is a Professor in the Department of Electrical and Computer Engineering, University of Wisconsin, Madison. He has spent research sabbaticals at the CEA Fontenay-aux-Roses, France, in 1970, and at the JET tokamak at Culham, England, in 1983. He has been active with graduate students and scientists in theoretical, computational, and experimental research in the areas of linear and nonlinear plasma waves, laser-formed plasma, and heating of fusion plasmas and lasers. He also teaches and carries out research in the areas of plasma RF sources for materials processing and Cerenkov amplifiers.

Dr. Scharer is a member of Tau Beta Pi, the American Physical Society, and the Center for Plasma Theory and Computation at the University of Wisconsin. 